\title{
A New Approach on Solving Intuitionistic Fuzzy Nonlinear Programming Problem
}

\author{
C. Loganathan ${ }^{1}$, M. Lalitha ${ }^{2 *}$ \\ ${ }^{1}$ Dept. of Mathematics, Maharaja Arts and Science College, Coimbatore, India \\ $2^{2 *}$ Dept. of Mathematics, Kongu Arts and Science College, Erode, India \\ *Corresponding Author: lali_math@rediffmail.com
}

Available online at: www.isroset.org

Received $06^{\text {th }}$ Sep 2017, Revised $14^{\text {th }}$ Sep 2017, Accepted $10^{\text {th }}$ Oct 2017, Online $30^{\text {th }}$ Oct 2017

\begin{abstract}
In this paper we propose an algorithm for solving Intuitionistic fuzzy nonlinear programming problems (IFNLPP) involving triangular Intuitionistic fuzzy numbers (TIFN). Here, the multi-objective nonlinear problem is converted into a single objective nonlinear programming problem and the problem is defuzzified by using triangular Intuitionistic fuzzy numbers. Then the problem is converted into crisp nonlinear programming problem. Numerical examples are provided to show the efficiency of the proposed algorithm.
\end{abstract}

Keywords- Intuitionistic fuzzy set, Triangular Intuitionistic fuzzy numbers, membership and non-membership value, Ambiguity and score value.

\section{INTRODUCTIONS}

Nonlinear programming is one of the most important operations research techniques. In an earlier work sanjaya Kumar Behera and Jyoti Ranjan Nayak [1] have proposed to find optimal solution to fuzzy nonlinear programming problems.Fuzzy sets are an efficient and reliable tool that allows us to handle such systems having imprecise parameters effectively. Atanossov [2] extended the fuzzy sets to the theory Intuitionistic fuzzy sets. His studies emphasized that in a view of handling imprecision, vagueness or uncertainty information both the degree of belonging and degree of non-belonging should be considered as two independent properties as these are not complement of each other. The concept of IFS can be viewed as an alternative approach to define a fuzzy set, in case where available information is not sufficient for the definition of an imprecise concept by means of a conventional fuzzy set. Thus, it is expected that IFS can be used to simulate human decision-making process and activities requiring human expertise and knowledge that are inevitably imprecise or totally reliable. Dubey et al [3] have studied with fuzzy linear programming with intuitionistic fuzzy numbers.

Recently, $\mathrm{Li}$ et al [4,5] has introduced a ratio ranking method for triangular intuitionistic fuzzy numbers. Then, it is applied to solve MADM problems. To this end, the value and ambiguity of TIFNs are used to obtain a new ranking approach. Using similar idea, Salahsshour et al [6] proposed other new ranking approach for TIFNs based on the value and ambiguity. Here, we have given some basic definitions and proposed an algorithm for solving Intuitionistic fuzzy nonlinear programming problems (IFNLPP) involving triangular Intuitionistic fuzzy numbers (TIFN).

The organization of the paper is as follows, Section I contains the introduction of Intuitionistic fuzzy nonlinear programming problems (IFNLPP) and triangular Intuitionistic fuzzy numbers, Section II contain the some basic definitions and new ranking functions are given, Section III contain the mathematical formulation of Intuitionistic fuzzy nonlinear programming problem, Section IV contain the proposed method to solve the Multiobjective Intuitionistic fuzzy nonlinear programming problem section $\mathrm{V}$ contain the numerical example by using proposed method, Section VI describes results and conclusion.

\section{Preliminaries}

Definition 2.1: [7]

Let $\mathrm{X}$ is a collection of objects denoted by $x$, and then a fuzzy set $\tilde{a}$ in $\mathrm{X}$ is a set of ordered pairs:

$\tilde{a}=\left\{\left(x, \mu_{\tilde{a}}(x) / x \in X\right\}\right.$, Where $\mu_{\tilde{a}}(x)$ is called the membership function of grade of membership of $x$ in $\tilde{a}$ that maps $X$ to the membership space $[0,1]$.

\section{Definition 2.2:}

Let $\mathrm{X}$ is a collection of objects then an intiuitionistic fuzzy set $\tilde{a}$ in $\mathrm{X}$ is defined as: $\tilde{a}=\left\{\left(x, \mu_{\tilde{a}}(x), v_{a}(x) / x \in X\right\}\right.$, where $\mu_{\tilde{a}}(x)$ and $v_{a}(x)$ are called the membership and nonmember ship functions of $x$ in $\tilde{a}$ respectively. 
Where $\mu_{a}: X \rightarrow[0,1] \quad$ and $\quad v_{a}: X \rightarrow[0,1]$ and $\mu_{\tilde{a}}(x)+v_{a}(x) \leq 1$

\section{Definition 2.3:}

For every common fuzzy subset $\tilde{a}$ on $\mathrm{X}$, Intiuitionistic Fuzzy Index of $\mathrm{X}$ in $\tilde{a}$ is defined as $\pi_{\tilde{a}}(x)=1-\mu_{\tilde{a}}(x)-v_{a}(x)$. It is also known as degree of hesitancy or degree of uncertainty of the element of $x$ in A. Obviously, for every $x \in X, 0 \leq \pi_{\tilde{a}}(x) \leq 1$.

\section{Definition 2.4:}

An Intiuitionistic Fuzzy Number (IFN) $\tilde{a}$ is an Intiuitionistic fuzzy subset of the real line.

(a) Normal, that is there is any $x_{0} \in R$, such that $\mu_{\tilde{a}}\left(x_{0}\right)=1, v_{a}\left(x_{0}\right)=0$.

(b) Convex for the membership function $\mu_{\tilde{a}}(x)$, that is,

$\mu_{\tilde{a}}\left(\lambda x_{1}+(1-\lambda) x_{2}\right) \geq \min \left(\mu_{\tilde{a}}\left(x_{1}\right), \mu_{\tilde{a}}\left(x_{2}\right)\right) \quad$ for every $x_{1}, x_{2} \in R, \lambda \in[0,1]$.

(c) Concave for the non-membership function $v_{\tilde{a}}\left(\lambda x_{1}+(1-\lambda) x_{2}\right) \leq \max \left(v_{\tilde{a}}\left(x_{1}\right), v_{\tilde{a}}\left(x_{2}\right)\right)$ for $\quad$ every $x_{1}, x_{2} \in R, \lambda \in[0,1]$.

Definition 2.5: [8]

$\tilde{a}$ is Triangular Intuitionistic Fuzzy Number (TIFN) with parameters $a_{1}^{\prime} \leq a_{1} \leq a_{2} \leq a_{3} \leq a_{3}^{\prime}$ and denoted by $\tilde{a}=\left(a_{1}, a_{2}, a_{3}, w_{\tilde{a}} ; a_{1}^{\prime}, a_{2}, a_{3}^{\prime}, u_{\tilde{a}}\right)$ having the membership function and non-membership function as follows:

$$
\mu_{\tilde{a}}(x)=\left\{\begin{array}{ll}
0 & \text { for } x \leq a_{1} \\
\frac{x-a_{1}}{a_{2}-a_{1}} & \text { for } a_{1} \leq x \leq a_{2} \\
1 & \text { for } x=a_{2} \\
\frac{a_{3}-x}{a_{3}-a_{2}} & \text { for } a_{2} \leq x \leq a_{3} \\
0 & \text { for } x>a_{3}
\end{array}\right\}
$$

$$
\mu_{\tilde{a}}(x)=\left\{\begin{array}{ll}
1 & \text { for } x<a_{1} \\
\frac{a_{2}-x}{a_{2}-a_{1}} & \text { for } a_{1} \leq x \leq a_{2} \\
0 & \text { for } x=a_{2} \\
\frac{x-a_{2}}{a_{3}-a_{2}} & \text { for } a_{2} \leq x \leq a_{3} \\
1 & \text { for } x>a_{3}
\end{array}\right\}
$$

Note:

Here $\mu_{\tilde{a}}(x)$ increases with constant rate for $x \in\left[a_{1}, a_{2}\right]$ and decreases with constant rate for $x \in\left[a_{2}, a_{3}\right]$, but $v_{a}(x)$ decreases with constant rate for $x \in\left[a_{1}^{\prime}, a_{2}\right]$ and increases with constant rate for $x \in\left[a_{2}, a_{3}^{\prime}\right]$.

\section{Particular cases}

Let $\tilde{a}=\left(a_{1}, a_{2}, a_{3}, w_{\tilde{a}} ; a_{1}, a_{2}, a_{3}, u_{\tilde{a}}\right)$ be a Triangular Intuitionistic Fuzzy Number. Then the following cases aries

\section{Case 1:}

If $a_{1}^{\prime}=a_{1}, a_{3}=a_{3}^{\prime}$ then $\tilde{a}$ represent Triangular Fuzzy Number (TFN).

\section{Case 2:}

If $a_{1}^{\prime}=a_{1}=a_{3}=a_{3}^{\prime}=\mathrm{m}$ then $\tilde{a}$ represent a real number $\mathrm{m}$.

We denote this triangular Intuitionistic fuzzy number by $\tilde{a}=\left(a_{1}, a_{2}, a_{3}, w_{\tilde{a}} ; a_{1}^{\prime}, a_{2}, a_{3}^{\prime}, u_{\tilde{a}}\right)$. We use $\mathrm{F}(\mathrm{R})$ to denote the set of all Intuitionistic fuzzy numbers.

Also if $m=a_{2}$ represents the modal value (or) midpoint, $\alpha_{1}=\left(a_{2}-a_{1}\right) \quad$ represents the left spread and $\beta_{1}=\left(a_{3}-a_{2}\right)$ right spread of membership function and $\alpha_{2}=\left(a_{2}-a_{1}\right) \quad$ represents the left spread and $\beta_{2}=\left(a_{3}-a_{2}\right)$ right spread of non-membership function.

Definition 2.6: [9]

and 
Let $\tilde{a}=\left(a_{1}, a_{2}, a_{3}, w_{\tilde{a}} ; a_{1}^{\prime}, a_{2}, a_{3}^{\prime}, u_{\tilde{a}}\right)$ be a Triangular Intuitionistic Fuzzy Number. Then the value and ambiguity of $\tilde{a}$ are given as follows.

(i) The value of the membership function of $\tilde{a}_{\text {is, }}$,

$$
v_{\mu}(\tilde{a})=\frac{\left(a_{1}+4 a_{2}+a_{3}\right) w_{\tilde{a}}}{6}
$$

While the value of the non- membership function is,

$$
v_{v}(\tilde{a})=\frac{\left(a_{1}^{\prime}+4 a_{2}+a_{3}^{\prime}\right)\left(1-u_{\tilde{a}}\right)}{6}
$$

(ii) The ambiguity of the membership function of $\tilde{a}$ is,

$$
B_{\mu}(\tilde{a})=\frac{\left(a_{3}-a_{1}\right) w_{\tilde{a}}}{3}
$$

While the ambiguity of the non- membership function is,

$$
B_{v}(\tilde{a})=\frac{\left(a_{3}^{\prime}-a_{1}^{\prime}\right)\left(1-u_{\tilde{a}}\right)}{3}
$$

\section{FUZZY INTUITIONISTIC NONLINEAR Programming Problem: $[10,11]$}

Intuitionistic Nonlinear programming problem with triangular intuitionistic fuzzy variables is defined as follows:

$\operatorname{Max} \stackrel{\sim}{Z}=\sum_{j=1}^{m} \tilde{c}_{j} \stackrel{\sim I}{x}_{j}$

Subject to

$\sum_{j=1}^{m} \tilde{a}_{i j} \stackrel{\sim}{x}_{j} \leq \tilde{b}_{i}^{I}$ for all $\mathrm{i}=1,2, \ldots \ldots \ldots \ldots . .$.

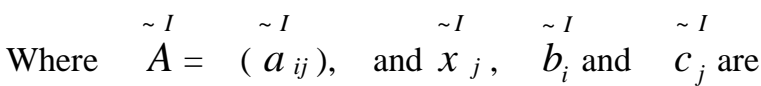

intuitionistic fuzzy numbers and $\tilde{x}_{j} \geq \tilde{0}$,

$\mathrm{j}=1,2 \ldots \mathrm{n}$.

\section{PROPOSED METHOD}

1. To find the solution of a multi-objective intuitionistic fuzzy nonlinear programming problem,

Define $\quad \tilde{a}=\left[\left(a_{1}, a_{2}, a_{3}, w_{\tilde{a}}\right),\left(a_{1}^{\prime}, a_{2}, a_{3}^{\prime}, u_{\tilde{a}}\right)\right] \quad$ and $\tilde{b}=\left[\left(b_{1}, b_{2}, b_{3}, w_{\tilde{b}}\right),\left(b_{1}^{\prime}, b_{2}, b_{3}^{\prime}, u_{\tilde{b}}\right)\right]$

2. Convert the multi-objective intuitionistic fuzzy nonlinear programming problem into a single objective intuitionistic fuzzy nonlinear programming problem using $\tilde{a}+\tilde{b}=\left\{\left(a_{1}+b_{1}, a_{2}+b_{2}, a_{3}+b_{3} ; \min \left\{w_{\tilde{a}}, w_{\tilde{b}}\right\}\right)\right\}$,

$\left\{\left(a_{1}^{\prime}+b_{1}^{\prime}, a_{2}+b_{2}, a_{3}^{\prime}+b_{3}^{\prime} ; \min \left\{u_{\tilde{a}}, u_{\tilde{b}}\right\}\right)\right\}$

3. Defuzzify the IFNLP into a crisp nonlinear programming using

The value of $p(\tilde{a})$,

$p(\tilde{a})=S_{\mu}(\tilde{a})-S_{v}(\tilde{a})$

Where $S_{\mu}(\tilde{a})=\frac{V_{\mu}(\tilde{a})}{1+B_{\mu}(\tilde{a})}$

$S_{v}(\tilde{a})=\frac{V_{v}(\tilde{a})}{1+B_{v}(\tilde{a})}$

4. Formulate the nonlinear programming problem.

5. Solve the NLPP by any one of the used procedure.

\section{NUMERICAL EXAMPLE}

$\operatorname{Max} \tilde{5} x_{1}+\tilde{3} x_{2}-\tilde{6} x_{1}^{2}$

$\operatorname{Max} \tilde{25} x_{1}+\tilde{48} x_{2}-\tilde{12} x_{1}^{2}$

Subject to

$\tilde{4} x_{1}+\tilde{3} x_{2} \leq \tilde{12}$

$\tilde{1} x_{1}+\tilde{3} x_{2} \leq \tilde{6}$

$x_{1}, x_{2} \geq 0$

Where,

$C_{1}=\widetilde{5}=\{(4,5,6 ; 3 / 4)(4,5,6.1 ; 1 / 4)\}$

$C_{2}=\widetilde{3}=\{(2.5,3,3.2 ; 1 / 2)(2,3,3.5 ; 1 / 4)\}$

$C_{3}=\tilde{6}=\{(5.5,6,7.5 ; 3 / 4)(5,6,8.1 ; 1 / 4)\}$

$P_{1}=\tilde{25}=\{(19,25,33 ; 0.9)(18,25,34 ; 1)\}$ 


$$
\begin{aligned}
& P_{2}=\tilde{48}=\{(44,48,54 ; 0.9)(43,48,56 ; 1)\} \\
& P_{3}=\tilde{12}=\{(11,12,13 ; 1)(11,12,14 ; 0)\}
\end{aligned}
$$

Subject to

$$
\begin{aligned}
& a_{11}=\tilde{4}=\{(3,5,4.1 ; 1)(3,4,5 ; 0)\} \\
& a_{12}=\tilde{3}=\{(2.5,3,3.5 ; 3 / 4)(2.4,3,3.6 ; 1 / 5)\} \\
& a_{21}=\tilde{1}=\{(0,1,2 ; 1)(0,1,2 ; 0)\} \\
& a_{22}=\tilde{3}=\{(2.8,3,3.2 ; 3 / 4)(2.5,3,3.2 ; 1 / 6)\} \\
& b_{1}=\tilde{12}=\{(11,12,13 ; 1)(11,12,14 ; 0)\} \\
& b_{2}=\tilde{6}=\{(5.5,6,7.5 ; 3 / 4)(5,6,8.1 ; 1 / 4)\}
\end{aligned}
$$

For, converting multi-objective into single objective,

$$
\begin{aligned}
& X_{1}=C_{1}+P_{1} \\
& \quad=\{(4+19,5+25,6+33 ; \min \{3 / 4,0.9\}) \\
& (4+18,5+25,6.1+34 ; \max \{1 / 4,1\})\} \\
& \quad=\{(23,30,39 ; 3 / 4)(22,30,40.1 ; 1)\}
\end{aligned}
$$

Similarly,

$$
\begin{aligned}
X_{2} & =C_{2}+P_{2} \\
& =\{(46.5,51,57.2 ; 0.5)(45,51,59.5 ; 1)\} \\
X_{3} & =C_{3}+P_{3} \\
& =\{(16.5,18,20.5 ; 3 / 4)(16,18,22.1 ; 1 / 4)\}
\end{aligned}
$$

For Defuzzification,

$$
\begin{aligned}
& X_{1}=\{(23,30,39 ; 3 / 4)(22,30,40.1 ; 1)\} \\
& V_{\mu}(\tilde{a})=22.75 \quad, V_{v}(\tilde{a})=0, \quad B_{\mu}(\tilde{a})=4, \\
& B_{v}(\tilde{a})=0, S_{\mu}(\tilde{a})=4.55 S_{v}(\tilde{a})=0
\end{aligned}
$$

Therefore,
$\mathrm{P}\left(\mathrm{X}_{1}\right)=4.55$

Similarly,

$$
\begin{aligned}
& \mathrm{P}\left(\mathrm{X}_{2}\right)=9.2137 \\
& \mathrm{P}\left(\mathrm{X}_{3}\right)=1.362 \\
& \mathrm{P}\left(\mathrm{a}_{11}\right)=0.8775 \\
& \mathrm{P}\left(\mathrm{a}_{12}\right)=-0.018 \\
& \mathrm{P}\left(\mathrm{a}_{21}\right)=0 \\
& \mathrm{P}\left(\mathrm{a}_{22}\right)=0.005 \\
& \mathrm{P}\left(\mathrm{b}_{1}\right)=3.14755 \\
& \mathrm{P}\left(\mathrm{b}_{2}\right)=0.47073
\end{aligned}
$$

Therefore the crisp nonlinear programming is

$$
\operatorname{Max} 4.55 x_{1}+9.2137-1.362 x_{1}^{2}
$$

Subject to

$$
\begin{aligned}
& 0.8775 x_{1}-0.018 x_{2} \leq 3.14755 \\
& 0 x_{1}+0.005 x_{2} \leq 0.47033 \\
& x_{1}, x_{2} \geq 0
\end{aligned}
$$

Using wolf's method, the optimum feasible solution is obtained and is given by

$$
x_{1}=1.670, x_{2}=94, \operatorname{Max} z=869.89
$$

\section{CONCLUSION}

We have proposed a new method to solve the multiobjective intuitionistic fuzzy nonlinear

Programming problem. This paper is useful to solve the multi-objective intuitionistic fuzzy nonlinear programming problem. It is also helps to defuzzification of TIFNLP problems. A numerical example is given to show the efficiency of the methodology.

\section{REFERENCES}

[1] sanjaya Kumar Behera., Jyoti Ranjan Nayak., Solutions of MultiObective Programmig Problems in Fuzzy approach, International Journal of Computer Science and Engineering, Vol.3, Issue 12, pp. 3790-3799, 2011.

[2] K.T Atanassov, Intuitionistic fuzzy sets, Fuzzy Sets and Systems, Vol.20, pp. 87-96, 1986. 
[3] Dipti Dubey., Aparna Mehra., Linear Programming with Triangular Intuitionistic Fuzzy Number, Advances in Intelligent Systems research, Vol.1, pp. 563-569, 2011.

[4] D.F Li, J.X Nan, and M.J Zhang, A ranking method of triangular intuitionistic fuzzy numbers and application to decision making, International Journal of Computational Intelligence Systems, Vol.3, Issue 5, pp.522-530, 2010.

[5] Deng-Feng-Li, A ratio ranking method of Triangular Intuitionic fuzzy number and its Application to MADM problems, computers Mathematics with applications, Vol.60, pp.1557-1570, 2010.

[6] S.Salahshour, G.A Shekari, A. Hakimzadeh, A Novel approach Ranking triangular Intuitionistic fuzzy numbers, AWER Proedia Information Technology, Vol.1, pp.442-446, 2012.

[7] L.A Zadeh, Fuzzy sets Information and Control, Vol.8, pp. 338 353, 1969.

[8] P. Burillo, H. Bustince, V. Mohedano, Some definitions of intuitionistic fuzzy number. First Properties; the Proceedings of the 1st Workshop on Fuzzy Based Expert Systems, 1994.

[9] J.Q Wang, Z. Zhang, Multi-criteria decision-making method with incomplete certain Information based on intuitionistic fuzzy number, Control and Decision, Vol.24, Issue 2, pp.226-230, 2009.

[10] V.L.G Nayagam, G.Venkateshwari and G.Sivaraman, Ranking of intuitionistic fuzzy numbers, IEEE International Conference, pp.1971-1974, 2008.

[11] Palash Dutta, Hrishikesh Boruah, Tazid Ali, Fuzzy Arithmetic with and without using $\alpha$-cut method: A comparative study, International Journal of Latest trends in Computing, Vol.2, Issue.1, pp. 99-107, 2011. 